\title{
Among the two kinds of metacognitive evaluation, only one is predictive of illusory object perception
}

Sandra Vetik ${ }^{\S}$, Kadi Tulver*, Diana Lints ${ }^{\S}$, Talis Bachmann ${ }^{\S}$

*University of Tartu, Estonia, Institute of Psychology; correspondence: sandravetik(at)gmail.com

$\S^{\S}$ University of Tartu, Estonia, School of Law

Abstract. The relationship between expectation-induced hallucination proneness and selfconfidence in performance was studied in a visual perception task. Participants were prompted either to recognize briefly shown faces as male or female or to rate the subjective vividness of a square surrounding the face. Importantly, in a few critical trials the square was absent. Upon completion, participants rated their performance in the face recognition task; they were also asked whether they were sure that their estimation was correct. Out of 35 participants, 33 "hallucinated" on at least one trial, rating the square as visible when it was actually absent. Negative correlation between hallucination proneness and self-confidence in performance (metacognitive rating) was found: the more hallucinations a participant experienced, the less confident (s)he was in his/her performance in the face recognition task. Most subjects underestimated their performance; higher ratings were also more accurate. Thus, higher hallucination proneness was associated with more inaccurate ratings of one's own perception. However, confidence in self-ratings as measured by the second follow-up question was unrelated to both, hallucination proneness and self-confidence in performance, supporting the view that there is no unitary mechanism of metacognitive evaluations and extending this view to the domain of visual hallucinatory perception.

Keywords: visual perception, metacognition, self-evaluation, illusion, hallucination, confidence, expectation

\section{Introduction}

Behavior is regulated not only by how environmental cues are perceived, but also by how the perceiver self-evaluates his/her perception in terms of reliability. Cognitive processes pertaining to this domain are subsumed under the concept of metacognition (Bang, Shekhar, \& Rahnev, 2018; Flavell, 1979; Koriat, 2012; Metcalfe, \& Shimamura, 1994; Yeung \& Summerfield, 2012). Generic descriptions of metacognitive processes refer to ,thinking about thinking“, „thinking about awareness“ (e.g., higher-order thought theory of consciousness - Rosenthal, 2005), „being aware of one's awareness“, „knowing about knowing“ etc.

In cognitive psychology and psychophysics of perception and memory an important distinction 
separates sensitivity (measured by objective correctness of detection, discrimination or recognition as corrected for guessing) and metacognitive evaluation of how confident a person is about the veridicality of his/her behavioral reports concerning the stimuli (de Gardelle \& Mamassian, 2014; Rahnev \& Fleming, 2019; Shea \& Frith, 2019; Spence, Dux, \& Arnold, 2016; Zehetleitner \& Rausch, 2013). It has been shown that the connection between objective behavioral sensitivity and subjective self-evaluation of it as measured by confidence ratings is by no means straightforwardly monotonic and/or linear and that our knowledge about this connection is far from definite (Fleming et al., 2010; Hanks \& Summerfield, 2017; Nauser et al., 2017; Koriat, 2012, 2018; Rahnev \& Denison, 2018; Rosenthal, 2019; Spence, Mattingley, \& Dux, 2018; Summerfield \& Tsetsos, 2015; Woodhead \& Baddeley, 1981).

Self-evaluations of performance operationalized by confidence measurements can be grouped into two general subtypes: evaluations of the efficacy or correctness of the ongoing or just completed tasks and evaluation of one's own capability for some cognitive function, including the ability to self-evaluate (Burns, Burns, \& Ward, 2016; Kleitman \& Stankov, 2007; Stankov, Pallier, Danthiir, \& Morony, 2012; see also Rosenberg, Schooler, Schoenbach, \& Rosenberg, 1995, on global vs specific self-esteem, and Fleming and Daw, 2017, on first-order and secondorder models of self-evaluation). However, virtually all studies devoted to investigation of these varieties of confidence evaluation have used tasks of perception, memory or problem-solving where the subjective experience refers to real, actually presented stimulation. We do not know of metacognitive confidence studies focused on so-called hallucinatory experiences where people experience objects that are not actually present (e.g., Aru \& Bachmann, 2017a; Aru, Tulver, \& Bachmann, 2018; McKenzie et al., 2010; Partos et al., 2016; Powers, Mathys, \& Corlett, 2017; Tompkins, Woods, \& Aimola-Davies, 2016).

This direction of research seems timely as hallucinations-including when observed in the neurotypical population - are purportedly explained in the context of the currently prevailing predictive coding approach. Predictive coding models of perception suggest that perception is determined by the relative weighting of sensory data and expectations (Friston, 2005; Hohwy, 2013; Clark, 2015, O'Callaghan et al., 2017). Depending on this relative weighting, the subjective perception depends more on actual sensory evidence or on prior knowledge; prior knowledge dominates perception when sensory data is degraded (Aru et al., 2012; 2016; Powers et al., 2017) or ambiguous (Sterzer, Frith, \& Petrovic, 2008; Weilnhammer et al., 2018) or when there is a strong prior (Kuhn \& Rensink, 2016; Tompkins et al., 2016). Hallucinatory experiences speak about a disbalance between the effects of priors and actual sensory inflow data processing. While subjective clarity of perception-like hallucinations have been studied (Aru \& Bachmann, 2017; Aru et al., 2018) by subjective scales such as PAS (Overgaard et al, 2006), metacognitive confidence in perceptual experiences occasionally intermingled with hallucinatory experiences has not been studied. To begin with, an exploratory study would be conceivable.

We formulated three hypotheses. The first hypothesis assumes that illusory perception 
exemplified by expectation based hallucinations has no correlation with confidence. (This is despite the fact that objective correctness of perception of actually presented stimuli is positively correlated with confidence in the clearly above-threshold range of objective performance - e.g., Bonder \& Gopher, 2019; Fleming et al., 2010; Rahnev \& Fleming, 2019; Rausch \& Zehetleitner, 2018; Shen \& Ma, 2019; however, see McIntosh et al., 2019, and Koriat, 2018.) This hypothesis has two sources. First, as the research on metacognitive beliefs in patients prone to positive symptoms has produced inconsistent results (see Moritz et al, 2010; Mintz \& Alpert, 1972), we opted for a null hypothesis. Second, there is some data showing that metacognitive differences (including self-certainty) do not correlate with positive symptoms like hallucination proneness in psychosis prone samples (Cotter et al, 2017; Moritz et al, 2016; Wright et al., 2020).

The second hypothesis assumed that the higher one's self-evaluation of his/her performance in the perceptual task, the higher one's confidence in the correctness of this self-evaluation. This hypothesis was put forth based on common intuition and some data about a general selfconfidence factor predictable by accuracy of performance in a variety of perceptual and other tasks (Bonder \& Gopher, 2019; Burns, Burns, \& Ward, 2016; Faivre et al., 2018; Kleitman \& Stankov, 2007). We predicted a connection between the subjective estimate of the level of correctness in the perceptual task and the level of general self-confidence about how reliable these former estimates were: we assumed that a person is more likely to be unsure of his/her performance estimation when the estimated level of performance was low, whereas confidence in self-evaluation increases with the estimated level of performance in the perception task.

The aim of the third hypothesis was to see whether the results of our research extend the finding of Woodhead and Baddeley (1981, cited according to Baddeley, 2013, pp 184-185, about there being no correlation between a person's opinion about the dependability of own memory and objectively correct memory) to perceptual tasks involving hallucinatory experiences.

\section{Method}

\subsection{Apparatus and participants}

For the computer-controlled experimental display a SUN CM751U CRT monitor with $1024 \mathrm{x}$ 768 pixels resolution and $100-\mathrm{Hz}$ refresh rate was used. The experiments were programmed and run using custom made Python scripts. In all experiments the observers viewed the arrays from a distance of $80 \mathrm{~cm}$.

Thirty five participants $(25 \mathrm{~F}, 10 \mathrm{M})$ took part in the experiment, their ages ranging from 18 to $62(\mathrm{M}=25.9)$. The sample size was determined by practical constraints. All participants had normal or corrected to normal vision. The study was approved by the ethics committee of University of Tartu and the experiment was carried out in compliance with national legislation and the Declaration of Helsinki. 


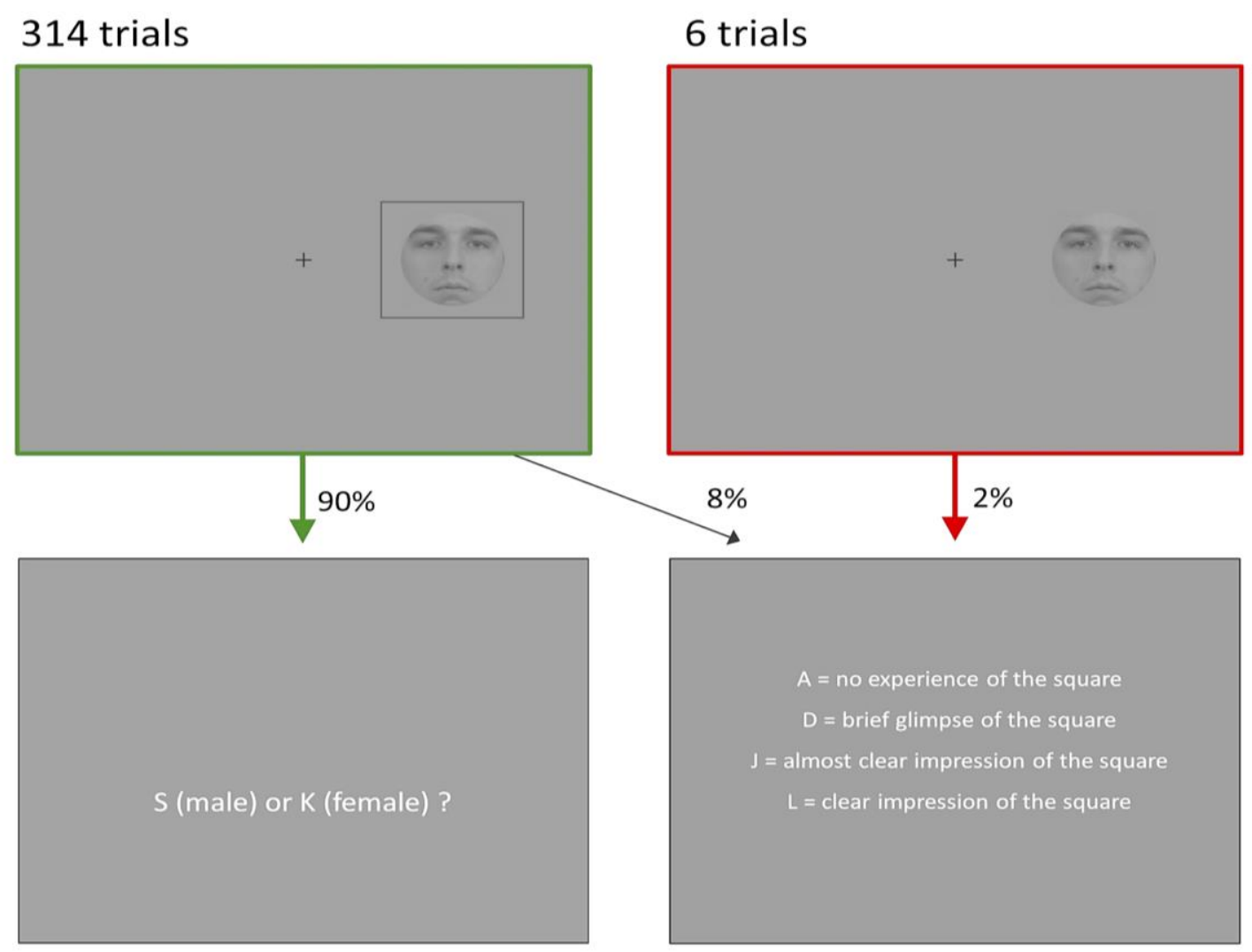

Figure 1. Illustration of the stimulus and response screens used in the task.

\subsection{Perception task}

The main experiment consisted of four blocks of 80 trials (altogether 320 trials). Each trial started with a fixation cross presented in the center of a grey screen for 1 second, followed by an endogenous-type arrow cue pointing to the left or right of the screen for $150 \mathrm{~ms}$. The cue was valid for $80 \%$ of the trials and invalid for $20 \%$. The stimulus was a peripherally presented male or female face with a neutral expression (1.8 degrees of visual angle), surrounded by the lines of a square-shaped figure (4 degrees of visual angle) slightly darker than the background (see Figure 1). The stimulus was presented on the left or right side of the screen for $100 \mathrm{~ms}$, followed by an empty screen for $700 \mathrm{~ms}$. The participants were instructed to fixate on the small cross in the center of the screen and direct their attention to the cued location. The average luminance of the background of visual stimuli was $15.8 \mathrm{~cd} / \mathrm{m}^{2}$.

On $90 \%$ of the trials, the task was to discriminate the gender of the face (male or female). The response keys were $\mathrm{S}$ (male) and $\mathrm{K}$ (female). On 10\% of the trials the participants' task was to 
rate how clearly they perceived the square on a four-point Perceptual Awareness Scale (PAS) (Overgaard et al, 2006). Here the response keys were A, D, J, L, where "1" corresponded to "no experience of the stimulus," "2" corresponded to "brief glimpse of the stimulus but could not recognize what it was," "3" corresponded to an "almost clear impression of the stimulus," and " 4 " corresponded to a "clear impression of the stimulus."

Before starting the main experiment, participants practiced both tasks separately for 30 trials each. The contrast of the square was varied only during the practice trials of the visibility task, to ensure that participants were using the subjective visibility scale as intended. In the main task, the luminosity of the background was $14.9 \mathrm{~cd} / \mathrm{m}^{2}$ and that of the square was $12.5 \mathrm{~cd} / \mathrm{m}^{2}$ (Michelson contrast 0.138).

On six critical trials (three in the valid and three in the invalid cue condition) no square was presented, although participants were asked to give a perceptual clarity rating. No critical trials were included for the first 80 trials, to ensure that the participants were already used to the task (i.e. that they had, by default, built up an expectation about the stimuli appearing on the screen).

\subsection{Metacognitive confidence}

Following the experiment, participants were given two multiple-choice questions. First, they were asked to rate their performance in the face recognition task by indicating on a 10-point scale (from $0-10 \%$ to $90-100 \%$ ) roughly what percentage of the faces they believed to have identified correctly. Secondly, they were asked whether they were certain in their self-rating, to which they could answer either "yes" or "no".

\subsection{Data analyses}

In the current study, the tendency for illusory perception was operationalized as the clarity with which participants perceived the missing squares in the critical trials. For each participant, average clarity rating across the six critical trials was calculated and used in data analyses as the illusory perception score. We also conducted the analyses using the number of trials in which the participant rated the square as clearly or almost clearly visible as an indicator of illusory perception, and obtained very similar results.

Participants' self-rating of performance was used as the metacognitive confidence score for their perceptual task performance. The self-rating was measured by a range of $10 \%$ steps $(0-10 \%, 10$ $20 \%$, etc); for the metacognitive confidence score, the midpoint of a given range was used (for example, for $20-30 \%$ it was $25 \%$ ).

For each participant, we also calculated a score reflecting the accuracy of his or her self-rating, by subtracting the metacognitive confidence score from the actual percentage of correct answers obtained in the perception task. The larger the score, the bigger the difference between self-rating 
and actual performance and thus the less accurate the self-rating.

Data was analysed using $\mathrm{R}$ software. The main analyses were performed using Pearson's correlation analyses and paired t-tests, supplemented by ANOVA.

Here, a remark is necessary for why signal detection theory (SDT, e.g., Macmillan \& Creelman, 2004) and/or type-2 signal detection theory (e.g., Morales, Odegaard, \& Maniscalso, 2019) based analyses of sensitivity and bias were not used. Essentially, this is predetermined by the ambiguity of what must be considered signal-in-noise if we are measuring subjective experiences of a nonpresented stimulus (Bachmann, 2004; see also Abid, 2019, and Rausch and colleagues, 2018, on related matters). Technically, this can be subsumed either under the category of misses (the sensory reality with a missing stimulus in it is missed) or category of false alarms (reporting a stimulus where there was none). Similarly, a "detected" (i.e., "hallucinated"), but actually nonpresent signal could be categorized as noise that makes an obstacle for correct detection of nonpresence of the signal. This also means that invoking the distinction between primary and secondary perception (Luccio, 2019) in an attempt to restore credibility of SDT under the "massive attack" from Bayesian approaches does not help much.

\section{Results}

Confirming the suitability of the procedure for obtaining illusory perception of actually absent visual stimulation, 33 out of 35 participants rated the square as visible in at least one of the critical trials, using a clarity rating of 2 (,,brief glimpse of the stimulus but could not recognize what it was") or more. 26 participants used a clarity rating of 3 ("almost clear impression of the stimulus") or 4 ("clear impression of the stimulus") and 18 participants used a rating of 4 at least once. 17 participants reported illusory perception ("hallucination" of the square) in more than 3 trials, of whom 7 experienced illusory perception in all six critical trials.

We analysed whether the validity of the cue had an effect on the subjective clarity rating of the square in critical and non-critical trials. We observed a significant main effect of trial type (critical/non-critical) on visibility ratings $(\mathrm{F}(1,34)=34.52$, $\mathrm{p}<.001$, ges $=0.407)$, but neither an effect of cue condition nor an interaction $(\mathrm{p}>.05)$.

Reaction time did not differ significantly between the critical and non-critical trials, although there was a trend showing faster responding in critical trials $(\mathrm{t}=-1.822, \mathrm{p}=.07)$. Reaction time in critical trials was unrelated to hallucination proneness $(r=-0.03, p=0.87)$.

We observed a significant positive correlation between the average clarity rating in critical trials and the average clarity rating in non-critical trials $(\mathrm{r}=0.6, \mathrm{p}<.001)$. In other words, participants who perceived the square more clearly when it was present also gave higher clarity ratings when it was absent. The average clarity rating in critical trials was lower than that in the non-critical trials $(\mathrm{t}=-3.74, \mathrm{p}<0.001)$. 
There was a significant negative correlation between hallucination proneness, as measured by the average clarity rating in the six critical trials, and self-confidence in performance, as measured by the self-rating ( $\mathrm{r}=-0.57, \mathrm{p}<.001)$ (Figure 2). The more clearly a subject "perceived" nonexisting squares, the lower he/she rated his/her performance in the face recognition task. (Wheter this may be a result of more attention divided to the square surrounding the face to the detriment of perception of face itself remains out of the scope of the present study.)

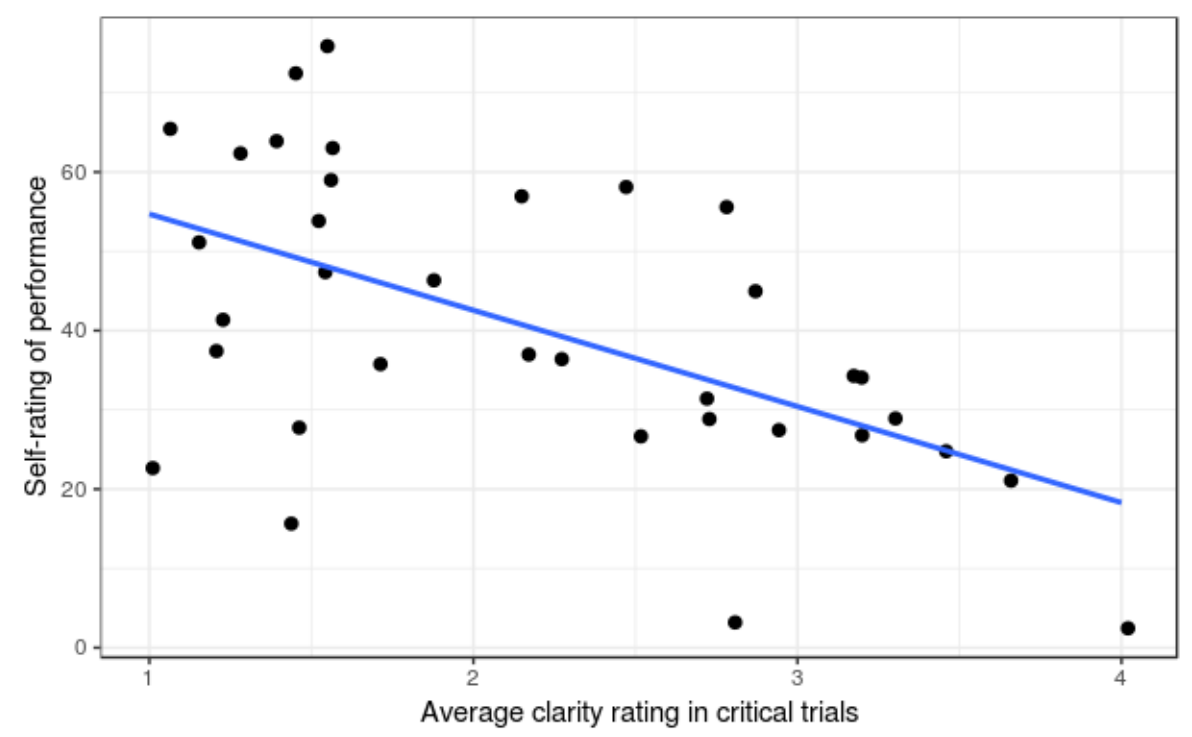

Figure 2. Scatterplot of the correlation between clarity ratings of the actually absent stimulus and selfratings of the correctness of one's own performance in the face discrimination task. Subjects more prone to experience hallucinations were less confident in their perception accuracy.

Most subjects underestimated their performance, making higher ratings more accurate. A significant positive correlation was found between hallucination proneness and the inaccuracy of self-rating $(\mathrm{r}=0.55, \mathrm{p}<.001)$ : people with higher hallucination proneness had more inaccurate ratings of their own conscious perception.

The actual percentage of correct answers was weakly correlated with the self-rating of performance $(\mathrm{r}=0.38, \mathrm{p}=.02)$. No association was found between confidence in self-rating, as measured by the second follow-up question, and hallucination proneness $(\mathrm{t}=-0.13, \mathrm{p}=.9)$. There was also no association between the two questions measuring self-confidence $(t=0.08, \mathrm{p}=.94)$.

\section{Discussion}

Our results show that the veridicality of perception depends not only on how accurately one perceives actually presented visual objects, but also on whether perception of objects not actually present - i.e., a hallucinatory experience - has occurred. This means that when conscious perception is measured by some visibility scales and/or confidence ratings together with measures of objective performance, subjective evaluations and objective measures may depart 
not only because actual stimuli were misperceived, but also because absent stimulation was mistakenly experienced as involving some "phantom" object(s). The overwhelming majority of our participants experienced at least some hallucinations as assessed by the PAS scale. Lack of behavioral sensitivity of a scale at its near-threshold end may stem from such illusory experiences. We recommend that experiments in this domain always include catch trials with absent stimuli in order to tease out the contribution of illusory/hallucinatory experiences in the results (Aru \& Bachmann, 2017a; Aru, Tulver, \& Bachmann, 2018; Bachmann, 2018). For models of consciousness mechanisms to be realistic they must also model the emergence of hallucinations.

While almost all subjects experienced illusory perception, the critical trials differed from noncritical trials in some ways. Firstly, although below the significance margin, reaction times tended to be faster in critical trials. This excludes the possibility that evaluations of the contents of these trials were based on some delayed working-memory processes possibly prone to confabulation. Secondly, actual stimuli were reported as having been perceived more clearly than were the clarity ratings in trials where subjects reported illusory perception. Therefore, we can infer that when illusory perception did occur, it was a different qualitative experience compared to the perception of an actual stimulus. One possibility to explain this observation stems from the microgenetic approach (Aru \& Bachmann, 2017b; Bachmann, 2000). According to the microgenetic stance, conscious experience emerges gradually, whereby the clarity and detailedness of the experienced object increases over time within about 100-300 ms. Faster reactions and lower clarity in hallucinated trials may mean that when an expected object is hallucinated, its microgenetic cycle is "frozen" at an early stage with vague representational content (e.g., proto-object or "blob" stage without fine detail). This allows also fast responses. In trials with real objects experienced, microgenetic process will go further from the proto-object (indistinct) stage up to the fine-grain representation. As this process takes longer and ends with more detailed experience, the clarity rating is higher and in some cases the response also has longer latency.

We formulated three hypotheses about illusory (i.e., hallucinatory) and veridical experiences for the present study. The first hypothesis assumed that perception of actually absent stimuli has no correlation with confidence in perception. However, our research revealed a strong correlation and the first hypothesis was overthrown -- illusory perception was negatively correlated with confidence relating to task performance (type-1 confidence). In other words, participants who were less likely to perceive absent stimuli gave more confident self-ratings of performance. According to the microgenetic theory this result can be expected if we assume that less distinct experiences in relatively frequent hallucinatory trials (due to the immature end of the microgenetic episode) offer more instances of under-articulated criterion contents for metacognitive evaluation. This leads to less confidence. On the other hand, for perceivers who rarely hallucinate, the comparative quality of perceptual experience of the actual stimulus and experience of the "empty" display location where the stimulus could be expected but is not 
present, is better distinguishable (i.e., without vague intermediate clarity stages). This in turn feeds confidence in visual discrimination. The latter assumption is supported by the well-known regularity whereby behavioral perceptual accuracy positively correlates with metacognitive confidence about task performance (Bonder \& Gopher, 2019; Fleming et al., 2010; Rahnev \& Fleming, 2019; Rausch \& Zehetleitner, 2018; Shen \& Ma, 2019).

There was no relationship between reaction time and level of illusory perception, meaning that the participants who gave higher clarity ratings did not differ from the participants who gave lower clarity ratings in terms of response speed. However, it cannot be ruled out that other types of response biases may have influenced the results. Subjects who tended to give higher clarity ratings in critical trials did so in non-critical trials as well. The relationship between high clarity ratings of illusory experiences and a low confidence rating might thus reflect an extreme response bias - that is, the tendency to answer in the extreme - of some subjects.

On the other hand, the consistency of clarity ratings could also mean that people who perceive existing objects more intensely experience more intense illusory perception as well. Research does seem to point to a strong association between mental imagery and perception, at least in the visual domain. It has been shown that visual imagery can have a functional effect on sensory processing akin to a weak form of visual perception, for example facilitating the perception of a relevant stimulus (Pearson, 2019; Tartaglia et al., 2009). In addition, a lot of brain-imaging evidence demonstrates considerable overlap between brain areas involved in perception and imagery (Kosslyn \& Thompson, 2003; St-Laurent, Abdi, \& Buchsbaum, 2015; Todd et al, 2013; Todd et al, 2015); and experienced imagery vividness correlates with neural overlap between imagery and perception in the entire visual system (Dijkstra, Bosch, \& van Gerven, 2019). If we consider expectation based hallucinations as a form of imagery, it is natural to expect that a trait of relatively stronger involvement of imagery mechanisms also predicts stronger involvement of direct perception serving mechanisms. (However, see Fulford et al, 2018, on the possibility that perception and imagery mechanisms may in some ways work in opposite direction.)

In the current experiment, more confident ratings were also more accurate. Thus, the relationship between confidence and hallucination proneness could in fact be explained by the relationship between accuracy and hallucination proneness. Mintz and Alpert (1972) found that subjects who experienced auditory hallucinations at a clinical level were less accurate in their self-ratings of auditory perception than healthy subjects or schizophrenic subjects who did not experience hallucinations, though they tended to overestimate their performance as opposed to the current study. In order to tease out the individual contributions of accuracy and confidence, future studies should use tasks that are subjectively perceived as relatively easier compared to the task in the current study.

The second hypothesis assumed that the higher the self-rating of task performance (type-1 confidence), the greater one's certainty in the self-rating (type-2 confidence). This hypothesis 
was also not confirmed. There was no association between the self-rating of task performance and confidence in one's evaluative performance. This shows that a person can be very certain that only a small part of his/her evaluation in a perceptual task is true. Conversely but similarly, a person can be uncertain whether his/her high self-rating of perception is accurate. In our research, the putative unique factor of self-evaluation as manifesting similarly in type-1 and type-2 confidence estimations was not supported. Metacognitive confidence seems to have different facets or subtypes depending on the self-dependent action for which its reliability or quality has to be evaluated. To put it in a different way - metacognitive evaluation of a subordinate metacognitive evaluation are not mutually predictive in terms of their level of confidence. On the other hand, if looked at from within the same level of metacognitive processes as directed at different tasks, commonality has been found. For example, Faivre and colleagues (2018) examined whether metacognition operates through shared, domain-general mechanisms, or through idiosyncratic, domain-specific mechanisms, but remained within the set of unimodal and intermodal perceptual tasks. They found that (i) metacognitive efficiency correlated between auditory, tactile, visual, and audiovisual tasks, (ii) confidence in an intermodal task was best modeled by supramodal formats assuming integrated intermodal representations, (iii) confidence in correct responses involved similar electrophysiological markers for different modal tasks. The common denominator was association with motor preparation preceding the perceptual judgment. Faivre et al. (2018) concluded that metacognitive domain-generality is based on supramodal confidence estimates and decisional signals shared across sensory modalities.

The aim of the third hypothesis was to see whether our results could extend the findings that there is no correlation between a person's confidence in a memory and the objective accuracy of a memory (Baddeley, 2013) to the case of direct perception (involving hallucinations). This hypothesis was confirmed. Neither type of confidence was related to actual performance in the perception task. This means that a very confident person can give false answers and the other way around.

For an interim summary, it appears that in subjects more prone to hallucinations we find less confidence in performance in a perception task involving the presentation of brief visual stimuli and that metacognitive evaluations of the accuracy of one's own perception are not significantly related to metacognitive evaluations of one's capacity for reliable type-1 evaluations.

The relationship between confidence and objectively measurable capability to give truthful reports about external stimulation is complicated and multifaceted (Abid, 2019; Burns, Burns, \& Ward, 2016; de Gardelle \& Mamassian, 2014; Fleming et al., 2010; Fleming \& Daw, 2017; Kleitmen \& Stankov, 2007; Koriat, 2012, 2018; McIntosh et al., 2019; Rahnev \& Denison, 2018; Rahnev \& Fleming, 2019; Rausch et al., 2018; Rosenthal, 2019; Shea \& Frith, 2019; Spence, Dux, \& Arnold, 2016; Spence, Mattingley, \& Dux, 2018; Stankov, Pallier, Danthiir, \& Morony, 2012; Summerfield \& Tsetsos, 2015; Woodhead \& Baddeley, 1981; Zehetleitner \& Rausch, 
2013). The results of the present study add more aspects to consider when examining relations between objective task performance, metacognitive self-evaluation of task performance and metacognitive self-evaluation of the capacity for reliable self-evaluation. More vivid nonveridical perceptual experiences ("hallucinations") caused by associatively formed task-specific contextual expectations may be predictive of lowered metacognitive confidence levels in a neurotypical sample, but only when it comes to metacognitive evaluation of task performance. Precision of metacognitive evaluation of the preciseness of first-order, task directed evaluation appears independent of the propensity to hallucinatory experiences. The relative weight of expectation-related priors compared to actual sensory evidence (possibly carrying the error signal) in percept formation is hypothetically higher in hallucination prone subjects. Correctness of responses in a perceptual task, on the contrary, requires better discrimination of actual stimulation and therefore a relatively weaker effect of prior expectations on each specific encounter with objects of perception. These considerations explain why propensity to hallucinate is related to objective perceptual task performance and its metacognitive evaluation. However, as the higher order metacognitive evaluation is not exclusively targeted at perceptual experience, but focuses on a non-perceptual aspect of behavior associated with self-referential and personality traits related dispositions, the link to first-order evaluation is lost. As a bold theoretical proposition, we could say that first-order metacognitive evaluations in perceptual decision making are dependent primarily on the access to phenomenal consciousness (Block, 1995) and on the learned associations of perceptual experience with objectively evidenced behavioral success, whereas second-order metacognitive evaluations depend on reference to selfconsciousness in its personality-trait aspect.

Our results also bear some applied aspects. For example, in a forensic context, exploring the confidence of a witness and the evidence in which a person is more or less confident can help evaluate the testimony and determine how to proceed with the investigation. Namely, ascertaining higher level metacognitive predispositions of an eyewitness may not be a good strategy, but assessing the metacognitive confidence with regard to a specific perceptual task performance could be of practical value. Of course, all conclusions made on the basis of the present research are obtained by examining a small group of people and this is a definite limitation of our study. In order to make generally sound conclusions there is a need to continue similar research with larger samples and a highly relevant variety of perceptual objects.

Acknowledgments. The present research benefited from institutional research support grant IUT20-40 (sub-account TSVPH14140I) from Estonian Science Agency. We are also grateful to Renate Rutiku and Carolina Murd for their assistance at some stages of this work.

\section{References}


1. Abid, G. (2019). Deflating inflation: the connection (or lack thereof) between decisional and metacognitive processes and visual phenomenology. Neuroscience of consciousness, 2019(1), niz015.

2. Aru, J., Axmacher, N., Do Lam, A. T., Fell, J., Elger, C. E., Singer, W., \& Melloni, L. (2012). Local category-specific gamma band responses in the visual cortex do not reflect conscious perception. Journal of Neuroscience, 32(43), 14909-14914.

3. Aru, J., \& Bachmann, T. (2017a). Expectation creates something out of nothing: The role of attention in iconic memory reconsidered. Consciousness and Cognition, 53, 203-210.

4. Aru, J., \& Bachmann, T. (2017b). In and out of consciousness: How does conscious processing (d)evolve over time? Frontiers in Psychology, 8:128. doi: 10.3389/fpsyg.2017.00128

5. Aru, J., Axmacher, N., Do Lam, A. T., Fell, J., Elger, C. E., Singer, W., \& Melloni, L. (2012). Local category-specific gamma band responses in the visual cortex do not reflect conscious perception. Journal of Neuroscience, 32(43), 14909-14914.

6. Aru, J., Rutiku, R., Wibral, M., Singer, W., \& Melloni, L. (2016). Early effects of previous experience on conscious perception. Neuroscience of consciousness, $2016(1)$, niw004.

7. Aru, J.,Tulver, K., \& Bachmann, T. (2018) It's all in your head: Expectations create illusory perception in a dual-task setup. Consciousness and Cognition, 65, 197-208.

8. Bachmann, T. (2000) Microgenetic approach to the conscious mind. Amsterdam/ Philadelphia: John Benjamins.

9. Bachmann, T. (2004). Inaptitude of the signal detection theory, useful vexation from the microgenetic view, and inevitability of neurobiological signatures in understanding perceptual (un)awareness. Consciousness and Cognition, 13(1), 101-106.

10. Bachmann, T. (2018). Excess of individual variability of priors prevents successful development of general models. Behavioral and Brain Sciences, 41, E224.

11. Baddeley, A. (2013). Essentials of human memory (classic edition). Taylor \& Francis / Psychology Press.

5. Bang, J. W., Shekhar, M., \& Rahnev, D. (2018). Sensory noise increases metacognitive efficiency. Journal of Experimetal Psychology: General, 148(3),437-452.

6. Block, N. (1995). On a confusion about a function of consciousness. Behavioral and brain sciences, 18(2), 227-247.

7. Bonder, T., \& Gopher, D. (2019). The effect of confidence rating on a primary visual task. Frontiers in Psychology, 10, 2674.

8. Burns, K. M., Burns, N. R., \& Ward, L. (2016). Confidence-More a Personality or Ability Trait? 
It Depends on How It Is Measured: A Comparison of Young and Older Adults. Frontiers in psychology, 7, 518. doi:10.3389/fpsyg.2016.00518.

9. Clark, A. (2015). Surfing uncertainty: Prediction, action, and the embodied mind. Oxford University Press.

10. Cotter, J., Yung, A. R., Carney, R., \& Drake, R. J. (2017). Metacognitive beliefs in the at-risk mental state: a systematic review and meta-analysis. Behaviour Research and Therapy, 90, 25-31.

11. de Gardelle, V., \& Summerfield, C. (2011). Robust averaging during perceptual judgment. Proceedings of the National Academy of Sciences of the United States of America, 108, 1334113346. http://dx.doi.org/10.1073/pnas.1104517108

12. Dijkstra, N., Bosch, S. E., \& van Gerven, M. A. J. (2019). Shared neural mechanisms of visual perception and imagery. Trends in Cognitive Sciences, 23, 423-434.

13. Faivre, N., Filevich, E., Solovey, G., Kühn, S., \& Blanke, O. (2018). Behavioral, Modeling, and Electrophysiological Evidence for Supramodality in Human Metacognition. The Journal of Neuroscience : the Official Journal of the Society for Neuroscience, 38(2), 263-277.

14. Fleming, S. M., \& Daw, N. D. (2017). Self-evaluation of decision-making: A general Bayesian framework for metacognitive computation. Psychological review, 124(1), 91-114.

15. Fleming, S. M., Whiteley, L., Hulme, O. J., Sahani, M., \& Dolan, R. J. (2010). Effects of category-specific costs on neural systems for perceptual decision-making. Journal of neurophysiology, 103(6), 3238-3247.

16. Friston, K. (2005). A theory of cortical responses. Philosophical transactions of the Royal Society B: Biological sciences, 360(1456), 815-836.

17. Fulford, J., Milton, F., Salas, D., Smith, A., Simler, A., Winlove, C., \& Zeman, A. (2018). The neural correlates of visual imagery vividness-An fMRI study and literature review. Cortex, 105, 26-40.

18. Hanks, T. D., \& Summerfield, C. (2017). Perceptual decision making in rodents, monkeys, and humans. Neuron, 93(1), 15-31.

19. Hauser, T. U., Allen, M., Rees, G., \& Dolan, R. J. (2017). Metacognitive impairments extend perceptual decision making weaknesses in compulsivity. Scientific Reports, 7(1), 6614.

20. Hohwy, J. (2013). The predictive mind. Oxford University Press.

21. Kleitman, S., \& Stankov, L. (2007). Self-confidence and metacognitive processes. Learning and Individual Differences, 17(2), 161-173.

22. Koriat, A. (2012). The self-consistency model of subjective confidence. Psychological Review, $119(1), 80-113$. 
23. Koriat, A. (2018). When reality is out of focus: Can people tell whether their beliefs and judgments are correct or wrong?. Journal of Experimental Psychology: General, 147(5), 613-631.

24. Kosslyn, S., \& Thompson, W. L. (2003). When is early visual cortex activated during visual mental imagery? Psychological Bulletin, 129,723-746.

25. Kuhn, G., \& Rensink, R. A. (2016). The Vanishing Ball Illusion: a new perspective on the perception of dynamic events. Cognition, 148, 64-70.

26. Luccio, R. (2019). Limits of the application of Bayesian modeling to perception. Perception, 48(10), 901-917.

27. Macmillan, N. A, \& Creelman, C. D. (2004). Detection theory: A user's guide. Mahwah, NJ: Lawrence Erlbaum.

28. McIntosh, R. D., Fowler, E. A., Lyu, T., \& Della Sala, S. (2019). Wise up: Clarifying the role of metacognition in the Dunning-Kruger effect. Journal of Experimental Psychology. General, 148(11), 1882-1897.

29. McKenzie, K. J., Poliakoff, E., Brown, R. J., \& Lloyd, D. M. (2010). Now you feel it, now you don't: How robust is the phenomenon of illusory tactile experience? Perception, 39(6), 839-850.

30. Metcalfe, J., \& Shimamura, A. P. (Eds.). (1994). Metacognition: Knowing about knowing. MIT press.

31. Mintz, S., \& Alpert, M. (1972). Imagery vividness, reality testing, and schizophrenic hallucinations. Journal of Abnormal Psychology, 79(3), 310-316.

32. Morales, J., Odegaard, B., \& Maniscalco, B. (2019). The Neural Substrates of Conscious Perception without Performance Confounds. In F. de Brigard, W. Sinnott-Armstrong (Eds.), Anthology of Neuroscience \& Philosophy.

33. Moritz, S., Balzan, R. P., Bohn, F., Veckenstedt, R., Kolbeck, K., Bierbrodt, J., \& Dietrichkeit, M. (2016). Subjective versus objective cognition: Evidence for poor metacognitive monitoring in schizophrenia. Schizophrenia Research, 178(1-3), 74-79.

34. Moritz, S., Peters, M. J., Larøi, F., \& Lincoln, T. M. (2010). Metacognitive beliefs in obsessivecompulsive patients: a comparison with healthy and schizophrenia participants. Cognitive Neuropsychiatry, 15(6), 531-548.

35. O’Callaghan, C., Kveraga, K., Shine, J. M., Adams Jr, R. B., \& Bar, M. (2017). Predictions penetrate perception: Converging insights from brain, behaviour and disorder. Consciousness and Cognition, 47, 63-74.

36. Overgaard, M., Rote, J., Mouridsen, K., \& Ramsøy, T. Z. (2006). Is conscious perception gradual or dichotomous? A comparison of report methodologies during a visual task. Consciousness and 
Cognition, 15(4), 700-708.

37. Partos, T. R., Cropper, S. J., \& Rawlings, D. (2016). You don't see what I see: Individual differences in the perception of meaning from visual stimuli. PloS one, 11(3), e0150615.

38. Pearson, J. (2019) The human imagination: the cognitive neuroscience of visual mental imagery. Nature Reviews Neuroscience, 20, 624-634. doi:10.1038/s41583-019-0202-9

39. Powers, A. R., Mathys, C., \& Corlett, P. R. (2017). Pavlovian conditioning-induced hallucinations result from overweighting of perceptual priors. Science, 357(6351), 596-600.

40. Rahnev, D., \& Denison, R. N. (2018). Suboptimality in perceptual decision making. Behavioral and Brain Sciences, 41(e223), 1-66.

41. Rahnev, D., \& Fleming, S. M. (2019). How experimental procedures influence estimates of metacognitive ability. Neuroscience of consciousness, 2019(1), niz009.

42. Rausch, M., Hellmann, S., \& Zehetleitner, M. (2018). Confidence in masked orientation judgments is informed by both evidence and visibility. Attention, Perception, \& Psychophysics, 80(1), 134-154.

43. Rausch, M., \& Zehetleitner, M. (2018). Multiple statistical signatures of human confidence. bioRxiv, 426635.

44. Rosenberg, M., Schooler, C., Schoenbach, C., \& Rosenberg, F. (1995). Global Self-Esteem and Specific Self-Esteem: Different Concepts, Different Outcomes. American Sociological Review, 60(1), 141-156.

45. Rosenthal, D. (2005). Consciousness and mind. Clarendon Press.

46. Rosenthal, D. (2019). Consciousness and confidence. Neuropsychologia, 128, 255-265.

47. Rouder, J. N., \& Haaf, J. M. (2018). A psychometrics of individual differences in experimental tasks. Psychonomic Bulletin \& Review, 1-16.

48. Shadlen, M. N., \& Kiani, R. (2013). Decision making as a window on cognition. Neuron, 80(3), 791-806.

49. Shea, N., \& Frith, C. D. (2019). The Global Workspace Needs Metacognition. Trends in Cognitive Sciences, 23(7), 560-571.

50. Shen, S., \& Ma, W. J. (2019). Variable precision in visual perception. Psychological Review, 126(1), 89-132.

51. Siegel, M., Engel, A. K., \& Donner, T. H. (2011). Cortical network dynamics of perceptual decision-making in the human brain. Frontiers in human neuroscience, 5, 21.

52. Spence, M. L., Dux, P. E., \& Arnold, D. H. (2016). Computations underlying confidence in visual perception. Journal of Experimental Psychology: Human Perception and Performance, 42(5), 
671-682.

53. Spence, M., Mattingley, J., \& Dux, P. (2018). Uncertainty Information That Is Irrelevant for Report Impacts Confidence Judgments. Journal of Experimental Psychology: Human Perception and Performance, 44(12), 1981-1994.

54. St-Laurent, M., Abdi, H., \& Buchsbaum, B. R. (2015). Distributed patterns of reactivation predict vividness of recollection. Journal of Cognitive Neuroscience, 27(10), 2000-2018.

55. Stankov, L., Pallier, G., Danthiir, V., \& Morony, S. (2012). Perceptual Underconfidence: A Conceptual Illusion?. European Journal of Psychological Assessment, 28(3), 190-200.

56. Sterzer, P., Frith, C., \& Petrovic, P. (2008). Believing is seeing: expectations alter visual awareness. Current Biology, 18(16), R697-R698.

57. Summerfield, C., \& Tsetsos, K. (2015). Do humans make good decisions? Trends in Cognitive Sciences, 19(1), 27-34.

58. Tartaglia, E. M., Bamert, L., Mast, F. W., \& Herzog, M. H. (2009). Human perceptual learning by mental imagery. Current Biology, 19(24), 2081-2085.

59. Todd, R. M., Schmitz, T. W., Susskind, J. \& Anderson, A. K. (2013). Shared neural substrates of emotionally enhanced perceptual and mnemonic vividness. Frontiers in Behavioral Neuroscience, 7:40. doi: 10.3389/fnbeh.2013.00040

60. Todd, R. M., Ehlers, M. R., Müller, D. J., Robertson, A., Palombo, D. J., Freeman, N., ... \& Anderson, A. K. (2015). Neurogenetic variations in norepinephrine availability enhance perceptual vividness. Journal of Neuroscience, 35(16), 6506-6516.

61. Tompkins, M. L., Woods, A. T., \& Aimola, A. D. (2016). The Phantom Vanish Magic Trick: Investigating the Disappearance of a Non-existent Object in a Dynamic Scene. Frontiers in psychology, 7, 950-950.

62. Weilnhammer, V. A., Stuke, H., Sterzer, P., \& Schmack, K. (2018). The neural correlates of hierarchical predictions for perceptual decisions. Journal of Neuroscience, 38(21), 5008-5021.

63. Woodhead, M. M., Baddeley, A. D., \& Simmonds, D. C. V. (1979). On training people to recognize faces. Ergonomics, 22, 333-343.

64. Wright, A., Nelson, B., Fowler, D., \& Greenwood, K. (2020). Perceptual biases and metacognition and their association with anomalous self experiences in first episode psychosis. Consciousness and Cognition, 77, 102847.

65. Yeung, N., \& Summerfield, C. (2012). Metacognition in human decision making: Confidence and error monitoring. Philosophical Transactions of the Royal Society of London Series B, Biological Sciences, 367,1310-1321. 
66. Zehetleitner, M., \& Rausch, M. (2013). Being confident without seeing: What subjective measures of consciousness are about? Attention, Perception, \& Psychophysics, 75, 1406-1426. 